\title{
Surfactant-alcohol experiments for dense non-aqueous phase liquid removal: a review
}

\begin{abstract}
The aim of this paper is to review and to summarize the existing laboratory experiment studies from other researchers regarding surfactant alcohol experiment for dense non-aqueous phase liquid (DNAPL) removal, their approach, method of measurement, factor consideration and their findings together with result discussion. This review includes the selection of surfactant-alcohol, their important characteristics in the remediation of DNAPL, the laboratory experimental setup using 2-D laboratory model and enhanced remediation of DNAPL from recent laboratory studies. It has been shown in the laboratory experiment studies that solubilization is the dominant removal process of DNAPL. After surfactant concentration reached the critical micelle concentration, interfacial tension between DNAPL, water and soil decreased. This has resulted in increasing of solubility and removal rate of DNAPL. Mobilization also takes place in the removal process. However, most of the laboratory experiments did not consider other factor such as soil permeability, soil texture, and interfacial tension between soil and DNAPL. Further studies of surfactant-alcohol flushing shall be considered in the near future.
\end{abstract}

Keyword: Non-aqueous phase liquid; Groundwater; Remediation; Laboratory model; Solubilization 